\section{APTITUD COMBINATORIA GENERAL Y ESPECÍFICA DEL CONTENIDO DE AZÚCARES EN MAÍCES CRIOLLOS ELOTEROS}

\section{GENERAL AND SPECIFIC COMBINING ABILITY OF SUGAR CONTENT IN CORN LANDRACES}

\author{
Bulmaro Coutiño Estrada ${ }^{*}$, Víctor A. Vidal \\ Martínez $^{2}$, Bertha Cruz Gómez ${ }^{3}$ y Carolina Cruz \\ Vázquez ${ }^{3}$
}

${ }^{1}$ Campo Experimental Centro de Chiapas, Instituto Nacional de Investigaciones Forestales, Agrícolas y Pecuarias (INIFAP). Km 3 Carretera Ocozocoautla-Cintalapa. Ocozocoautla, Chiapas, México. Tel y fax 01(968) 688-2918 Ext 107. ${ }^{2}$ Campo Experimental Santiago Ixcuintla, INIFAP. Santiago Ixcuintla, Nayarit. ${ }^{3}$ Facultad de Ciencias Agronómicas, Universidad Autónoma de Chiapas. Villaflores, Chiapas.

* Autor para correspondencia (coutino.bulmaro@inifap.gob.mx)

\section{RESUMEN}

Seis variedades criollas de maíz, sobresalientes por sus cualidades eloteras y sus 15 cruzas directas se evaluaron en dos localidades de Chiapas con objeto de conocer la aptitud combinatoria general y la específica del contenido de azúcares medida en ${ }^{\circ}$ Brix, así como la heterosis manifestada en las cruzas. Se encontró que sólo los efectos génicos aditivos fueron significativos $(P \leq 0.05)$, lo que indica que esta característica elotera puede ser mejorada en las variedades criollas de mayores efectos de aptitud combinatoria general mediante el uso de esquemas de selección recurrente para explotar la varianza aditiva presente. Las variedades progenitoras 'Campechano' y 'Paloma' tuvieron los mayores efectos de ACG (0.781 y 0.381), al producir los elotes más dulces con 13.1 y $12.9{ }^{\circ}$ Brix, respectivamente. Las cruzas tuvieron valores bajos de heterosis.

Palabras clave: Zea mays, selección recurrente, elotes, contenido de azúcares.

\section{SUMMARY}

Six open-pollinated corn (Zea mays L.) landraces outstanding for the quality of their sweet green ears, and their 15 direct crosses, were evaluated at two locations in Chiapas. General and specific combining ability for grain sugar content, measured in ${ }^{\circ} \mathrm{Brix}$, as well as heterosis of their crosses was evaluated. It was found that only additive gene effects were significant $(P \leq \mathbf{0 . 0 5})$. Thus this ear sweetness related trait could be improved in parental varieties having the largest general combining ability by using recurrent selec- tion methods that exploit the additive genetic variance. Parental varieties 'Campechano' and 'Paloma' had the highest values of GCA effects (0.781 and 0.581$)$ and produced the sweetest green ears with 13.1 and 12.9 ${ }^{\circ}$ Brix. All crosses showed low values of heterosis.

Index words: Zea mays, recurrent selection, green corn, sugar content.

\section{INTRODUCCIÓN}

En muchas regiones de México los agricultores que cultivan maíz (Zea mays L.) contribuyen a la conservación y generación de la diversidad genética in situ (Bommer, 1991) al seleccionar deliberadamente las semillas más favorables por sus diversas características, a través de las variantes que se han ido presentando por selección natural, mutación, introducción, recombinación y aislamiento.

Gran parte de la diversidad genética del maíz nativo de México aún se puede encontrar en los campos agrícolas en forma de variedades criollas, ya que sólo en $23 \%$ de la superficie sembrada con maíz se usa semilla mejorada (Polanco y Flores, 2008); además, los agricultores siguen identificando características favorables a sus necesidades y preferencias y combinando materiales genéticos de manera creativa para generar mayor variación (Louette y Smale, 1996).

Existen diversos maíces eloteros, pero el de mejor sabor es el maíz dulce (sweet corn) cultivado en Norteamérica, que difiere de los dentados o cristalinos por tener el gene mutante "Sugary 1" (su1), el cual causa que el endospermo del grano acumule cerca del doble de azúcares, comparado con un maíz normal (Schultheis, 1998; Tracy et al., 2006). En el noreste de México, las razas Dulcillo del Noroeste de grano rugoso, y el Elotero de Sinaloa son cultivadas para consumo en elote o tortillas por su delicioso sabor, debido a su alto contenido de azúcares (Guerrero et al., 2010; Salinas et al., 2003); en el Estado de Hidalgo los maíces criollos son preferidos por sus elotes dulces (Guemes-Vera et al., 2004); en el Estado de Guanajuato, las razas Elotes Cónicos y Elotes Occidentales de granos rojos y azules sobresalen por sus excelentes tortillas de buen color, suavidad y elevados contenidos de antocianinas (Vázquez et al., 2010).

En las áreas de riego de Chiapas se cultivan diversas variedades de maíz para ser comercializadas en elote (Turrent et al., 2004), aun cuando no fueron seleccionadas o formadas para ese fin (Mauricio et al., 2004). Los precios de un elote en el campo varían desde $\$ 1.00$ a $\$ 2.50$ (precio de enero a mayo del 2010), según la región y fecha de corte, por lo que para muchos productores es más benéfico producir elotes que producir grano, además 
de que después del corte de elotes comercializan el forraje verde para alimentación de ganado.

Actualmente, en Chiapas no se cultivan variedades mejoradas de maíz específicas para la producción de elote; los productores eloteros siembran semilla de variedades criollas, mejoradas o híbridos con características favorables para comercialización: como longitud del elote, número de hileras de granos y sabor dulce por un alto contenido de azúcares (hasta $13.8{ }^{\circ}$ Brix) (CoutiñoEstrada et al., 2010).

En los programas de mejoramiento genético es importante conocer el tipo de acción génica involucrada en la expresión de caracteres de interés económico para desarrollar nuevas variedades. Los análisis dialélicos constituyen una herramienta útil para caracterizar y estimar los parámetros genéticos de progenitores y sus cruzas, mediante su aptitud combinatoria general (ACG) y aptitud combinatoria específica (ACE), y permiten definir el método de mejoramiento genético más adecuado (Hallauer y Miranda, 1981), predecir cruzas superiores y seleccionar plantas individuales que combinen las mejores características de los progenitores.

El objetivo del presente trabajo fue conocer la ACG y la ACE del contenido de azúcares de elotes de un grupo sobresaliente de variedades criollas eloteras y sus cruzas, así como la heterosis con respecto a la media de los progenitores, a fin de reunir elementos para proponer un método eficiente de mejora para esta característica.

\section{MATERIALES Y MÉTODOS}

Se formaron cruzas dialélicas durante el ciclo agrícola de riego Otoño-Invierno 2006, en las que como progenitores se usaron seis variedades criollas de la raza Tuxpeño (Figura 1), raza de plantas altas, tardías y con excelente cobertura de mazorca, que previamente fueron identificadas como sobresalientes por sus elotes largos y dulces (Coutiño-Estrada et al., 2010). Tales variedades fueron colectadas en los municipios de Villaflores, Jiquipilas, Villacorzo y Ocozocoautla, en la región Central de Chiapas.
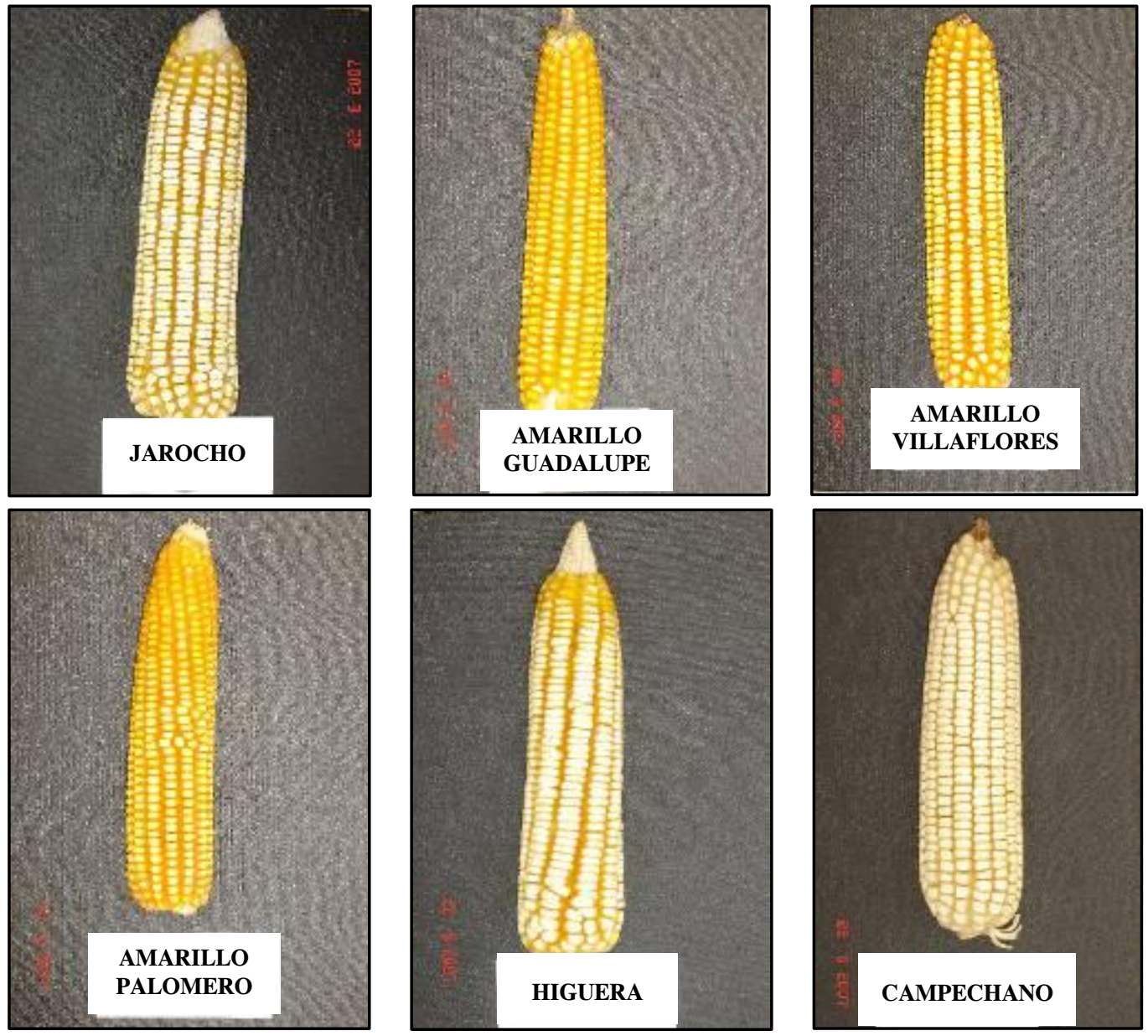

Figura 1. Variedades criollas eloteras progenitoras de las cruzas dialélicas. 
Durante el ciclo agrícola de temporal (secano) Primavera-Verano del 2007 las seis variedades progenitoras y sus 15 cruzas directas se evaluaron en dos ambientes, bajo un diseño experimental de bloques completos al azar con cuatro repeticiones, en donde la unidad experimental fue una parcela de dos surcos de $5 \mathrm{~m}$ de longitud, con 22 plantas por surco. El primer ambiente se ubicó en el municipio de Villaflores, a $15^{\circ} 14^{\prime} \mathrm{LN}$, $93^{\circ} 16^{\prime} \mathrm{LO}$ y una altitud de $610 \mathrm{~m}$. El segundo ambiente fue en el municipio de Ocozocoautla, Chiapas, a $16^{\circ} 45^{\prime}$ $\mathrm{LN}, 93^{\circ} 22^{\prime} \mathrm{LO}$, y una altitud de $800 \mathrm{~m}$.

El manejo del cultivo en ambos sitios fue el recomendado por el INIFAP, que incluye fertilizar el suelo con las dosis $150 \mathrm{~N}-60 \mathrm{P}-0 \mathrm{~K}$ y $120 \mathrm{~N}-40 \mathrm{P}-0 \mathrm{~K}$, para Vilaflores y Ocozocoautla, respectivamente; la primera aplicación fue con la mitad del nitrógeno (Urea) y todo el fósforo (Fosfato diamónico), y se hizo a las dos semanas posteriores a la siembra, y el resto del nitrógeno se aplicó a los 35 d de la siembra; ambas aplicaciones se hicieron en forma "mateada" (por planta). Cuando las plantas de todas las variedades estaban en la etapa de elote (90 d después de la siembra), de dos repeticiones se tomaron al azar cinco elotes en estado lechoso y sus granos fueron macerados para obtener un extracto y en él determinar los ${ }^{\circ}$ Brix, que miden la cantidad de sólidos solubles presentes; estos sólidos solubles están compuestos por los azúcares, ácidos, sales y demás compuestos solubles en agua. Para ello se utilizó un refractómetro digital Atago Pal-1® (Tokio, Japón), el cual proporciona las lecturas al colocar varias gotas del extracto del grano en el prisma.

Para el análisis estadístico se utilizó el programa computacional interactivo "Diallel" de la Estación Experimental de Lafayette, Louisiana, EE.UU (Magary y Kang, 1994). Este programa involucra a los progenitores junto con sus cruzas directas (Diseño Dialélico 2; Griffing, 1956) en varios ambientes, permite descomponer las sumas de cuadrados de los genotipos evaluados en los efectos de ACG de los progenitores y los de ACE de las cruzas, y proporciona la significancia de los mismos mediante la DMS al 0.05 de probabilidad de error. Con las medias de los genotipos se calculó el porcentaje de heterosis para cada cruza con base en el promedio de los dos progenitores involucrados.

\section{RESULTADOS Y DISCUSIÓN}

El coeficiente de variación obtenido fue de $8 \%$, que corresponde a un nivel aceptable de confiabilidad. En el análisis conjunto se detectaron diferencias significativas $(\mathrm{P} \leq 0.05)$ entre genotipos (progenitores y cruzas) para contenido de azúcares (Cuadro 1), lo que indica la existencia de variabilidad entre estos materiales criollos.
Al descomponer la variación genotípica en su aptitud combinatoria general y específica, los valores de ACG resultaron significativos, pero no los de ACE; es decir, estas características están determinadas principalmente por genes con efectos aditivos más que por genes con efectos de dominancia, lo que pudiera explicarse por una reducida diversidad genética entre las variedades criollas progenitoras, como se aprecia en la Figura 1, ya que todas ellas pertenecen a la raza Tuxpeño.

Cuadro 1. Cuadrados medios del análisis dialélico combinado a través de ambientes.

\begin{tabular}{lcc}
\hline Fuente de variación & Grados de libertad & ${ }^{\circ}$ Brix $^{1}$ \\
\hline Genotipos & 20 & $3.1293^{* *}$ \\
$\quad$ ACG & 5 & $8.4621^{* *}$ \\
$\quad$ ACE & 15 & $1.3517 \mathrm{~ns}$ \\
Ambientes & 1 & $119.2844 * *$ \\
Bloques x Amb. & 2 & \\
Genotipo x Amb. & 20 & $0.8770 \mathrm{~ns}$ \\
Error & 40 & 0.8242 \\
Total & 83 & \\
$*$ **: Significativo a 0.05 y 0.01 de probabilidad, respectivamente; ns \\
$=$ no significativo. ${ }^{1}$ Datos de dos repeticiones.
\end{tabular}

Los valores superiores $(\mathrm{P} \leq 0.05)$ de ACG para ${ }^{\circ}$ Brix se presentaron en los progenitores $\mathrm{P}_{6}$ 'Campechano' de Villacorzo (0.781) y $\mathrm{P}_{4}$ 'Paloma' de Jiquipilas (0.381). Según Sierra y Preciado (1988), a medida que la diversidad genética de los progenitores se incrementa, también aumenta la diferencia para los valores de aptitud combinatoria, ya sea para ACG o para ACE, o para los dos tipos de acción génica. Ello explicaría que una variedad chiapaneca de Jiquipilas y una introducida de Campeche tuvieran los valores más altos de ACG y produjeran una cruza superior a las demás con los elotes más dulces $\left(12.9{ }^{\circ} \mathrm{Brix}\right)$. Al respecto, Preciado et al. (2005) afirman que cuando se detectan efectos grandes de la aptitud combinatoria general, es factible explotar la proporción aditiva de la varianza genética disponible mediante cualquier variante de la selección recurrente. Entonces, estas dos variedades eloteras son candidatas a ser mejoradas para esta característica.

Entre ambientes también se encontraron diferencias (P $\leq 0.05)$ para ${ }^{\circ}$ Brix, ya que en Villaflores se produjeron elotes más dulces $\left(12.8^{\circ}\right.$ Brix) que en Ocozocoautla (10.1 ${ }^{\circ}$ Brix). Las condiciones ambientales durante el experimento no fueron completamente favorables, porque se presentó una temporada de huracanes y los fuertes vientos provocaron acame en algunas plantas ubicadas en Villaflores, lo que afectó su desarrollo y marcó diferencias notables entre ambos ambientes. 
Cuadro 2. Contenido de sólidos solubles (Grados Brix) de progenitores (en la diagonal) y cruzas (sobre la diagonal superior) y porcentaje de heterosis media (bajo la diagonal).

\begin{tabular}{lrrrrrr}
\hline & \multicolumn{5}{c}{ P r o g e n i t o r e s } \\
\cline { 2 - 7 } & \multicolumn{1}{c}{$\mathrm{P}_{1}$} & \multicolumn{1}{c}{$\mathrm{P}_{2}$} & $\mathrm{P}_{3}$ & $\mathrm{P}_{4}$ & $\mathrm{P}_{5}$ & $\mathrm{P}_{6}$ \\
\hline Progenitores y origen & 10.83 & $\mathbf{1 1 . 7 8}$ & 10.25 & 10.60 & 10.83 & $\mathbf{1 1 . 7 3}$ \\
P2 Amarillo de Guadalupe & 7.53 & 11.08 & 10.45 & 11.10 & 10.28 & $\mathbf{1 2 . 0 0}$ \\
P3 Amarillo de Villaflores & 1.01 & -7.21 & 11.50 & 10.60 & 10.93 & 11.90 \\
P4 Paloma de Jiquipilas & -10.97 & -7.3 & -12.84 & $\mathbf{1 2 . 8 8}$ & 11.10 & $\mathbf{1 2 . 9 0}$ \\
P5 Higuera de Ocozocoautla & 5.22 & -1.32 & 3.06 & -2.30 & 9.80 & 10.70 \\
P6 Campechano de Villacorzo & -1.88 & -0.62 & -3.36 & -0.96 & -6.68 & $\mathbf{1 3 . 1 0}$ \\
\hline
\end{tabular}

Números en negritas son estadísticamente iguales y superiores al resto $\left(\mathrm{DMS}_{0.05}=1.142\right)$.

En promedio, los maíces que produjeron los elotes más dulces fueron los progenitores $\mathrm{P}_{6}$ 'Campechano' (13.1 ${ }^{\circ}$ Brix) y $\mathrm{P}_{4}$ Paloma $\left(12.8{ }^{\circ}\right.$ Brix $)$, que estadísticamente fueron iguales a las cruzas $\mathrm{P}_{4} \times \mathrm{P}_{6}, \mathrm{P}_{2} \times \mathrm{P}_{6}$ y $\mathrm{P}_{1} \times \mathrm{P}_{6}$ (Cuadro 2). 'Campechano' también produjo los elotes más dulces en Villaflores (15.2 ${ }^{\circ}$ Brix). Los resultados de este estudio son similares a los de Coutiño-Estrada et al. (2010), quienes en 2006 evaluaron 40 maíces de la raza Tuxpeño en riego en Villaflores y Ocozocoautla, y encontraron que los elotes más dulces fueron los de los híbridos 'HV-521C', 'H-561', 'H-552C' y del criollo 'Amarillo de Villaflores', con 11.0, 10.3, 10.1 y 10.3 ${ }^{\circ}$ Brix, respectivamente; igualmente evaluaron 42 maíces de la raza Comiteco en condiciones de temporal en Teopisca, Chis., donde los más dulces fueron criollo 'Amarillo Bolita', 'V-231A', 'Teopisca-A C5' y 'Sintético-B', con 13.8, 12.9, 12.5 y 12.5 'Brix, respectivamente.

La mayor heterosis media fue obtenida en la cruza $\mathrm{P}_{1} \mathrm{x}$ $\mathrm{P}_{2}$ con $7.5 \%$. En el mejoramiento genético del rendimiento de grano se considera que el nivel deseable de heterosis para una cruza es de por lo menos $20 \%$ (Vasal y Córdova, 1996). Así, la escasa heterosis del contenido de azúcares encontrada en este estudio indica la carencia de diversidad genética entre las variedades criollas progenitoras, por lo que convendría combinarlas con germoplasma proveniente de regiones geográficas diferentes, pues a mayor divergencia genética se espera mayor heterosis (Gómez y Valdivia et al. 1988). Sin embargo la diversidad geográfica no siempre produce los resultados esperados, pues Gutiérrez et al. (2002) encontraron poca heterosis al combinar líneas de maíz de origen genético diverso. Los resultados aquí obtenidos permiten proponer que primero convendría mejorar las variedades criollas por métodos de selección recurrente, y posteriormente combinarlas con variedades de origen genético distante para así incrementar la heterosis en el dulzor del elote.

\section{CONCLUSIONES}

Se detectó variabilidad entre genotipos, atribuida principalmente a los efectos de ACG de las variedades progenitoras, que indica la presencia de acción génica aditiva en ellas. Las cruzas formadas entre las variedades criollas tuvieron valores bajos de heterosis para sólidos solubles totales. Las variedades 'Campechano' y 'Amarillo Paloma' mostraron los mayores valores de ACG. Dado que la acción génica aditiva fue significativa para el dulzor del elote, estas variedades criollas podrán ser mejoradas mediante el uso de esquemas de selección recurrente.

\section{BIBLIOGRAFÍA}

Bommer D F R (1991) The historical development of international collaboration in plant genetics resources. In: Crop Networks: Searching for New Concepts for Collaborative Genetic Resources. Th J L Van Hintun, L Frese, P M Perrer (eds). Papers of the EUCARPIA/IBPGR Symposium Held in Wageningen. The Netherlands. 3-6 December 1990. Inernational Crop Network Series No. 4 International Board for Plant Genetic Resources. Rome. pp:3-12.

Coutiño-Estrada B, V A Vidal-Martínez, G Sánchez-Grajalez (2010) Selección de maíces criollos con calidad elotera bajo condiciones de riego y temporal en Chiapas. In: Mejoramiento, Conservación y Uso de los Maíces Criollos. Nájera R M. B., C. A. Ramírez M (eds). Publicación Especial. Sociedad Mexicana de Fitogenética. Universidad Michoacana de San Nicolás de Hidalgo. México. D. F. pp:178-190.

Gómez M N, R Valdivia B (1988) Dialélico integrado con líneas de diferentes programas de maíz para la región cálida. Rev. Fitotec. Mex. 11:103-120.

Griffing B (1956) Concept of general and specific combining ability in relation to diallel crossing systems. Aust. J. Biol. Sci. 9:463493.

Guemes-Vera N, A D Hernández F, M I Reyes S, E N Aquino B, M E Espíndola, G Dávila O, E Mercado $S$ (2004) Caracterización física, química y estructural de 3 variedades de elotes de color de la región del Valle de Tulancingo, Hidalgo. In: Memorias VII Congreso Nacional de Ciencia de los Alimentos y III Foro de Ciencia y Tecnología de Alimentos. Guanajuato, Méx. pp:120-126.

Guerrero H M, A Ortega C, V A Vidal M, O Palacios V, O Cota A (2010) Diversidad y distribución de los maíces nativos en el Noroeste de México. In: Mejoramiento, Conservación y Uso de los Maíces Criollos. M B Nájera-Rincón, C A Ramírez M (eds). Publicación Especial. Sociedad Mexicana de 
Fitogenética. Universidad Michoacana de San Nicolás de Hidalgo. México. D. F. pp:119-129..

Gutiérrez D E, A Palomo G, A Espinosa B, E De la Cruz L (2002) Aptitud combinatoria y heterosis para rendimiento de líneas de maíz en la Comarca Lagunera. Rev. Fitotec. Mex. 25:271-277.

Hallauer A R, J B Miranda Fo (1981) Quantitative Genetics in Maize Breeding. Iowa State University Press. Ames, Iowa, USA. 468 p.

Louette D, M Smale (1996) Genetic diversity and maize seed management in a traditional Mexican community: implications for in situ conservation of maize. NRG Paper 96-03. CIMMYT. México D.F. 21 p.

Magary R, M S Kang (1994) Interactive BASIC Program for Griffing's Diallel Analyses. The J. Hered. 85:336.

Mauricio R S, J Figueroa C, S Taba, M Reyes V, F Sánchez R, A Mendoza G (2004) Caracterización de accesiones de maíz por calidad de grano y tortilla. Rev. Fitotec. Mex. 27:213-222.

Polanco J A, T Flores M (2008) Bases para una Política de I\&D e Innovación de la Cadena de Valor del Maíz. Foro Consultivo Científico y Tecnológico, A. C. México, D. F. 244 p.

Preciado O R E, A Terrón, N Gómez M, E Robledo G (2005) Componentes genéticos en poblaciones heteróticamente contrastantes de maíz de origen tropical y subtropical. Agron. Mesoam. 16:145-151.

Salinas M Y, F Martínez B, M Soto H, R Ortega P, J L Arellano V (2003) Efecto de la nixtamalización sobre las antocianinas del grano de maíces pigmentados. Agrociencia 37:617-628.
Schultheis J R (1998) Sweet Corn Production. Extension Horticultural Specialist. Department of Horticultural Science. North Carolina Cooperative Extension Service. North Carolina State University. Raleigh, N. C., USA.

Sierra M M, E R Preciado O (1988) Análisis e interpretación de cruzas dialélicas con germoplama tropical precoz de maíz. Rev. Fitotec. Mex. 11:92-102.

Tracy W, S Whitt, E S Buckler (2006) Recurrent Mutation and Genome Evolution: Example of Sugary 1 and the Origin of Sweet Maize. Crop Sci. 46:49-54.

Turrent F A, R Camas G, A López L, M Cantú A, J Ramírez S, J Medina M, A Palafox C (2004) Producción de maíz bajo riego en el Sur-Sureste de México. I. Análisis agronómico. Agric. Téc. Méx. 30:153-167.

Vasal S K, H Córdova (1996) Heterosis en maíz: acelerando la tecnología de híbridos de dos progenitores para el mundo en desarrollo. Curso Internacional de Actualización en Fitomejoramiento y Agricultura Sustentable. UAAAN. Buenavista, Saltillo, Coah. pp:32-54.

Vázquez-Carrillo G, Salinas-Moreno Y, J A Aguirre-Gómez, J L Pons-Hernández, L García-Leaños, M Segura-Nieto (2010) Calidad en grano y tortillas en maíces nativos del estado de Guanajuato, México. In: Mejoramiento, Conservación y Uso de los Maíces Criollos. M B Nájera-Rincón, C A Ramírez M (eds). Publicación Especial. Sociedad Mexicana de Fitogenética. Universidad Michoacana de San Nicolás de Hidalgo. México. D. F. pp:166-176. 